\title{
Chiral constituent quark model and the coupling strength of $\eta^{\prime}$
}

\author{
Harleen Dahiya ${ }^{a}$, Manmohan Gupta ${ }^{a}$ and J.M.S. Rana ${ }^{b}$ \\ ${ }^{a}$ Department of Physics (Centre of Advanced Study in Physics), Panjab University, Chandigarh-160 014, India. \\ ${ }^{b}$ Department of Physics, H.N.B. Garhwal University, SRT Campus, Badshahithaul(Tehri-Garhwal), India.
}

(November 10, 2018)

\begin{abstract}
Using the latest data pertaining to $\bar{u}-\bar{d}$ asymmetry and the spin polarization functions, detailed implications of the possible values of the coupling strength of the singlet Goldstone boson $\eta^{\prime}$ have been investigated in the $\chi \mathrm{CQM}$ with configuration mixing. Using $\Delta u, \Delta_{3}, \bar{u}-\bar{d}$ and $\bar{u} / \bar{d}$, the possible ranges of the coupling parameters $a, \alpha^{2} a, \beta^{2} a$ and $\zeta^{2} a$, representing respectively the probabilities of fluctuations to pions, $K, \eta$ and $\eta^{\prime}$, are shown to be $0.10 \lesssim a \lesssim 0.14,0.2 \lesssim \alpha \lesssim 0.5,0.2 \lesssim \beta \lesssim 0.7$ and $0.10 \lesssim|\zeta| \lesssim 0.70$. To further constrain the coupling strength of $\eta^{\prime}$, detailed fits have been obtained for spin polarization functions, quark distribution functions and baryon octet magnetic moments corresponding to the following sets of parameters: $a=0.1, \alpha=0.4, \beta=0.7,|\zeta|=0.65$ (Case I); $a=0.1, \alpha=0.4, \beta=0.6,|\zeta|=0.70$ (Case II); $a=0.14, \alpha=0.4, \beta=0.2, \zeta=0$ (Case III) and $a=0.13, \alpha=\beta=0.45,|\zeta|=0.10$ (Case IV). Case I represents the calculations where $a$ is fixed to be 0.1 , in accordance with earlier calculations, whereas other parameters are treated free and the Case IV represents our best fit. The fits clearly establish that a small non-zero value of the coupling of $\eta^{\prime}$ is preferred over the higher values of $\eta^{\prime}$ as well as when $\zeta=0$, the latter implying the absence of $\eta^{\prime}$ from the dynamics of $\chi \mathrm{CQM}$. Our best fit achieves an overall excellent fit to the data, in particular the fit for $\Delta u, \Delta d, \Delta_{8}$ as well as the magnetic moments $\mu_{n}, \mu_{\Sigma^{-}}, \mu_{\Sigma^{+}}$and $\mu_{\Xi^{-}}$ is almost perfect, the $\mu_{\Xi^{-}}$being a difficult case for most of the similar calculations.
\end{abstract}

The chiral constituent quark model $(\chi \mathrm{CQM})$, as formulated by Manohar and Georgi [1], has recently got good deal of attention [2-5] as it is successful in not only explaining the "proton spin crisis" [6] but is also able to account for the $\bar{u}-\bar{d}$ asymmetry [7-9], existence of significant strange quark content $\bar{s}$ in the nucleon, various quark flavor contributions to the proton spin [2], baryon magnetic moments [2,3] and hyperon $\beta$-decay parameters etc.. Recently, it has been shown that configuration mixing generated by spin-spin forces [10-12], known to be compatible with the $\chi \mathrm{CQM}[13-15]$, improves the predictions of $\chi \mathrm{CQM}$ regarding the quark distribution functions and the spin polarization functions [16]. Further, $\chi \mathrm{CQM}$ with configuration mixing (henceforth to be referred as $\chi \mathrm{CQM}_{\text {config }}$ ) when coupled with the quark sea polarization and orbital angular momentum (Cheng-Li mechanism [17]) as well as "confinement effects" is able to give an excellent fit [16] to the baryon magnetic moments and a perfect fit for the violation of Coleman Glashow sum rule [18].

The key to understand the "proton spin problem", in the $\chi$ CQM formalism [3], is the fluctuation process

$$
q^{ \pm} \rightarrow \mathrm{GB}+q^{\prime} \rightarrow\left(q \bar{q}^{\prime}\right)+q^{\prime} \mp,
$$

where GB represents the Goldstone boson and $q \bar{q}^{\prime}+q^{\prime}$ constitute the "quark sea" [3-5,16]. The effective Lagrangian describing interaction between quarks and a nonet of GBs, consisting of octet and a singlet, can be expressed as

$$
\mathcal{L}=g_{8} \overline{\mathbf{q}} \Phi \mathbf{q}+g_{1} \overline{\mathbf{q}} \frac{\eta^{\prime}}{\sqrt{3}} \mathbf{q}=g_{8} \overline{\mathbf{q}}\left(\Phi+\zeta \frac{\eta^{\prime}}{\sqrt{3}} I\right) \mathbf{q}=g_{8} \overline{\mathbf{q}}\left(\Phi^{\prime}\right) \mathbf{q}
$$

where $\zeta=g_{1} / g_{8}, g_{1}$ and $g_{8}$ are the coupling constants for the singlet and octet GBs, respectively, $I$ is the $3 \times 3$ identity matrix. The GB field which includes the octet and the singlet GBs is written as

$$
\Phi^{\prime}=\left(\begin{array}{ccc}
\frac{\pi^{0}}{\sqrt{2}}+\beta \frac{\eta}{\sqrt{6}}+\zeta \frac{\eta^{\prime}}{\sqrt{3}} & \pi^{+} & \alpha K^{+} \\
\pi^{-} & -\frac{\pi^{0}}{\sqrt{2}}+\beta \frac{\eta}{\sqrt{6}}+\zeta \frac{\eta^{\prime}}{\sqrt{3}} & \alpha K^{0} \\
\alpha K^{-} & \alpha \bar{K}^{0} & -\beta \frac{2 \eta}{\sqrt{6}}+\zeta \frac{\eta^{\prime}}{\sqrt{3}}
\end{array}\right) \text { and } \quad q=\left(\begin{array}{c}
u \\
d \\
s
\end{array}\right) \text {. }
$$

$\mathrm{SU}(3)$ symmetry breaking is introduced by considering $M_{s}>M_{u, d}$ as well as by considering the masses of GBs to be nondegenerate $\left(M_{K, \eta}>M_{\pi}\right)[4,5,17]$, whereas the axial $\mathrm{U}(1)$ breaking is introduced by $M_{\eta^{\prime}}>M_{K, \eta}[3-5,17]$. The 
parameter $a\left(=\left|g_{8}\right|^{2}\right)$ denotes the probability of chiral fluctuation $u(d) \rightarrow d(u)+\pi^{+(-)}$, whereas $\alpha^{2} a, \beta^{2} a$ and $\zeta^{2} a$ respectively denote the probabilities of fluctuations $u(d) \rightarrow s+K^{-(0)}, u(d, s) \rightarrow u(d, s)+\eta$, and $u(d, s) \rightarrow u(d, s)+\eta^{\prime}$.

The chiral structure of QCD is known to have intimate connection with the $\eta$ and $\eta^{\prime}$ dynamics [19]. Recently, in the context of $\chi \mathrm{CQM}$, Steven D. Bass [20] has reiterated in detail the deep relationship of the non-perturbative aspects of QCD, including gluon anomaly, and the comparatively large masses of the $\eta$ and $\eta^{\prime}$ mesons. In particular, he has emphasized that the gluon degrees of freedom mix with the flavor singlet Goldstone state to increase the masses of $\eta$ and $\eta^{\prime}$ through the Witten-Veneziano mass formula [21]. Similarly, as shown earlier by Ohta et al [22] and recently advocated by Cheng and $\mathrm{Li}[3]$ that $\eta^{\prime}$ could play an important role in the formulation of the $\chi \mathrm{CQM}$ as it may correspond to the non-planar contributions in the $1 / N_{c}$ expansion. On the other hand, it has recently been observed on phenomenological grounds [5] that the new measurement of both the $\bar{u} / \bar{d}$ asymmetry as well as $\bar{u}-\bar{d}$ asymmetry by the NuSea Collaboration [8] may not require substantial contribution of $\eta^{\prime}$. In this context, it therefore becomes interesting to understand the extent to which the contribution of $\eta^{\prime}$ is required in the $\chi \mathrm{CQM}$ thereby giving vital clues to the dynamics of non-perturbative regime of QCD.

The purpose of the present communication is to phenomenologically estimate the coupling strength of $\eta^{\prime}$ by carrying out a fine grained analysis of "proton spin problem" and related issues within $\chi \mathrm{CQM}_{\text {config }}$ by including the latest data. In particular, it would be interesting to fine tune the contribution of $\eta^{\prime}$ by studying its implications on spin polarization functions, baryon octet magnetic moments and the quark distribution functions.

The details of $\chi \mathrm{CQM}_{\text {config }}$ have already been discussed in Ref. [16], however to facilitate the discussion as well as for the sake of readability of the manuscript, some essential details of $\chi \mathrm{CQM}$ with configuration mixing have been presented in the sequel. The most general configuration mixing, generated by the chromodynamic spin-spin forces [10-12], in the case of octet baryons can be expressed as $[11,12,23]$

$$
|B\rangle=\left(\left|56,0^{+}\right\rangle_{N=0} \cos \theta+\left|56,0^{+}\right\rangle_{N=2} \sin \theta\right) \cos \phi+\left(\left|70,0^{+}\right\rangle_{N=2} \cos \theta^{\prime}+\left|70,2^{+}\right\rangle_{N=2} \sin \theta^{\prime}\right) \sin \phi,
$$

where $\phi$ represents the $|56\rangle-|70\rangle$ mixing, $\theta$ and $\theta^{\prime}$ respectively correspond to the mixing among $\left|56,0^{+}\right\rangle_{N=0}-$ $\left|56,0^{+}\right\rangle_{N=2}$ states and $\left|70,0^{+}\right\rangle_{N=2}-\left|70,2^{+}\right\rangle_{N=2}$ states. For the present purpose, it is adequate [12,16,24] to consider the mixing only between $\left|56,0^{+}\right\rangle_{N=0}$ and the $\left|70,0^{+}\right\rangle_{N=2}$ states and the corresponding "mixed" octet of baryons is expressed as

$$
|B\rangle \equiv\left|8, \frac{1}{2}^{+}\right\rangle=\cos \phi\left|56,0^{+}\right\rangle_{N=0}+\sin \phi\left|70,0^{+}\right\rangle_{N=2},
$$

for details of the spin, isospin and spatial parts of the wavefunction, we refer the reader to reference [25].

To study the variation of the $\chi \mathrm{CQM}$ parameters and the role of the coupling strength of $\eta^{\prime}$ in obtaining the fit, one needs to formulate the experimentally measurable quantities having implications for these parameters as well as dependent on the unpolarized quark distribution functions and the spin polarization functions. We first calculate the spin polarizations and the related quantities which are affected by the "mixed" nucleon. The spin structure of a nucleon is defined as $[3-5,16]$

$$
\hat{B} \equiv\langle B|N| B\rangle,
$$

where $|B\rangle$ is the nucleon wavefunction defined in Eq. (5) and $N$ is the number operator given by

$$
N=n_{u^{+}} u^{+}+n_{u^{-}} u^{-}+n_{d^{+}} d^{+}+n_{d^{-}} d^{-}+n_{s^{+}} s^{+}+n_{s^{-}} s^{-},
$$

where $n_{q^{ \pm}}$are the number of $q^{ \pm}$quarks. The spin structure of the "mixed" nucleon, defined through the Eq. (5), is given by

$$
\left\langle 8, \frac{1}{2}^{+}|N| 8, \frac{1}{2}^{+}\right\rangle=\cos ^{2} \phi\left\langle 56,0^{+}|N| 56,0^{+}\right\rangle+\sin ^{2} \phi\left\langle 70,0^{+}|N| 70,0^{+}\right\rangle .
$$

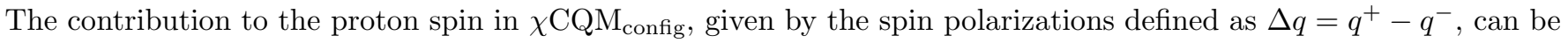
written as 


$$
\begin{gathered}
\Delta u=\cos ^{2} \phi\left[\frac{4}{3}-\frac{a}{3}\left(7+4 \alpha^{2}+\frac{4}{3} \beta^{2}+\frac{8}{3} \zeta^{2}\right)\right]+\sin ^{2} \phi\left[\frac{2}{3}-\frac{a}{3}\left(5+2 \alpha^{2}+\frac{2}{3} \beta^{2}+\frac{4}{3} \zeta^{2}\right)\right] \\
\Delta d=\cos ^{2} \phi\left[-\frac{1}{3}-\frac{a}{3}\left(2-\alpha^{2}-\frac{1}{3} \beta^{2}-\frac{2}{3} \zeta^{2}\right)\right]+\sin ^{2} \phi\left[\frac{1}{3}-\frac{a}{3}\left(4+\alpha^{2}+\frac{1}{3} \beta^{2}+\frac{2}{3} \zeta^{2}\right)\right] \\
\Delta s=-a \alpha^{2} .
\end{gathered}
$$

After having formulated the spin polarizations of various quarks, we consider several measured quantities which are expressed in terms of the above mentioned spin polarization functions. The flavor non-singlet components $\Delta_{3}$ and $\Delta_{8}$, usually calculated in the $\chi \mathrm{CQM}$, obtained from the neutron $\beta$-decay and the weak decays of hyperons are respectively,

$$
\begin{array}{r}
\Delta_{3}=\Delta u-\Delta d=-\frac{1}{9}\left(5 \cos ^{2} \phi+\sin ^{2} \phi\right)\left(-3+a\left(3+3 \alpha^{2}+\beta^{2}+2 \zeta^{2}\right)\right) \\
\Delta_{8}=\Delta u+\Delta d-2 \Delta s=-\frac{1}{3}\left(-3+a\left(9-3 \alpha^{2}+\beta^{2}+2 \zeta^{2}\right)\right) .
\end{array}
$$

The flavor non-singlet component $\Delta_{3}$ is related to the well known Bjorken sum rule [26]. Another quantity which is usually evaluated is the flavor singlet component related to the total quark spin content as

$$
\Delta \Sigma=\frac{1}{2}(\Delta u+\Delta d+\Delta s)=-\frac{1}{6}\left(-3+a\left(9+6 \alpha^{2}+\beta^{2}+2 \zeta^{2}\right)\right),
$$

in the $\Delta s=0$ limit, this reduces to the Ellis-Jaffe sum rule [27].

Apart from the above mentioned spin polarization we have also considered the quark distribution functions which have implications for $\zeta$ as well as for other $\chi \mathrm{CQM}$ parameters. For example, the antiquark flavor contents of the "quark sea" can be expressed as $[3-5,16]$

$$
\bar{u}=\frac{1}{12}\left[(2 \zeta+\beta+1)^{2}+20\right] a, \quad \bar{d}=\frac{1}{12}\left[(2 \zeta+\beta-1)^{2}+32\right] a, \quad \bar{s}=\frac{1}{3}\left[(\zeta-\beta)^{2}+9 \alpha^{2}\right] a,
$$

and

$$
u-\bar{u}=2, \quad d-\bar{d}=1, \quad s-\bar{s}=0 .
$$

The deviation of Gottfried sum rule [9], related to the $\bar{u}(x)$ and $\bar{d}(x)$ quark distributions, is expressed as

$$
I_{G}=\frac{1}{3}+\frac{2}{3} \int_{0}^{1}[\bar{u}(x)-\bar{d}(x)] d x=0.254 \pm 0.005 .
$$

In terms of the symmetry breaking parameters $a, \beta$ and $\zeta$, this deviation is given as

$$
\left[I_{G}-\frac{1}{3}\right]=\frac{2}{3}\left[\frac{a}{3}(2 \zeta+\beta-3)\right] \text {. }
$$

Similarly, $\bar{u} / \bar{d}[8,28]$ measured through the ratio of muon pair production cross sections $\sigma_{p p}$ and $\sigma_{p n}$, is expressed in the present case as follows

$$
\bar{u} / \bar{d}=\frac{(2 \zeta+\beta+1)^{2}+20}{(2 \zeta+\beta-1)^{2}+32} .
$$

Some of the other important quantities depending on the quark distribution functions which are usually discussed in the literature are the fractions of the quark content and are defined as follows

$$
f_{q}=\frac{q+\bar{q}}{\left[\sum_{q}(q+\bar{q})\right]}, \quad f_{3}=f_{u}-f_{d}, \quad f_{8}=f_{u}+f_{d}-2 f_{s} .
$$


Before discussing the results, we first discuss the general constraints on the $\chi \mathrm{CQM}_{\text {config }}$ parameters due to the spin polarization functions and quark distribution functions. The $\chi \mathrm{CQM}_{\text {config }}$ involves five parameters, $a, \alpha, \beta, \zeta$ and $\phi$, the mixing angle $\phi$ is fixed from the consideration of neutron charge radius $[12,23,29]$ as was done in our earlier calculations [16]. In the $\chi \mathrm{CQM}$, there is a broad consensus [3-5,16] about the parameters $a, \alpha^{2} a, \beta^{2} a$ and $\zeta^{2} a$, representing the probabilities of fluctuations to pions, $K, \eta$ and $\eta^{\prime}$, following the hierarchy $a>\alpha^{2} a>\beta^{2} a>\zeta^{2} a$. The parameter $\zeta$, representing the contribution of the singlet GB $\eta^{\prime}$ in the $\chi \mathrm{CQM}$, as well as the parameters $a, \alpha$ and $\beta$ cannot be fixed independently from the spin polarization functions and quark distribution functions. To begin with, we have carried out a broader analysis wherein we have attempted to find the ranges of the $\chi \mathrm{CQM}$ parameters from $\Delta u, \Delta_{3}, \bar{u}-\bar{d}, \bar{u} / \bar{d}$.

The range of the coupling breaking parameter $a$ can be easily found by considering the expression of the spin polarization function $\Delta u$ (Eq. (9)), by giving the full variation of parameters $\alpha, \beta$ and $\zeta$ from which one finds $0.10 \lesssim a \lesssim 0.14$, in agreement with the values considered in other similar calculations $[3-5,16]$. The range of the parameter $\zeta$ can be found from the expression of $\bar{u} / \bar{d}$ (Eq. (19)) which involves only $\beta$ and $\zeta$. Using the possible range of $\beta$, i.e. $0<\beta<1$ as well as the latest experimental measurement of $\bar{u} / \bar{d}[8]$, one finds $-0.70 \lesssim \zeta \lesssim-0.10$. The parameter $\zeta$, except in the quark distribution functions, occurs as $\zeta^{2}$ in the spin polarization functions and the calculations of spin dependent quantities, therefore, for the purpose of discussion, the constraint on $\zeta$ can be expressed as $0.10 \lesssim|\zeta| \lesssim 0.70$. The range of $\beta$ can be found by using the $\bar{u}-\bar{d}$ asymmetry representing the violation of Gottfried sum rule [9]. In terms of the $\chi \mathrm{CQM}$ parameters, the $\bar{u}-\bar{d}$ asymmetry can be expressed as

$$
\bar{u}-\bar{d}=\frac{a}{3}(2 \zeta+\beta-3) .
$$

Using the above found ranges of $a$ and $\zeta$ as well as the latest measurement of $\bar{u}-\bar{d}$ asymmetry [8], $\beta$ comes out to be in the range $0.2 \lesssim \beta \lesssim 0.7$. Similarly, the range of $\alpha$ can be found by considering the flavor non-singlet component $\Delta_{3}(=\Delta u-\Delta d)$ and it comes out to be $0.2 \lesssim \alpha \lesssim 0.5$.

Our gross analysis as well as earlier analyses $[3-5,16]$ brings out that $a$, representing the "pion" fluctuation, expectedly is the most important parameter. The parameters $\alpha$ and $\beta$ are nearly equal whereas the parameter $\zeta$ is strongly coupled to $a$ through the $\bar{u}-\bar{d}$ asymmetry,. After finding the ranges of the coupling breaking parameters, we have carried out a fine grained analysis, first by fitting $\Delta u, \Delta d$ and $\Delta_{3}[30]$ and then calculating the other spin polarization functions, quark distribution functions and baryon octet magnetic moments with the same values of parameters. The analysis consist of several fits which have been carried out to constrain the coupling strength of $\eta^{\prime}$.

In several previous calculations the parameter $a$ has usually been fixed to be $0.1[3-5,16]$, therefore we have also attempted to fit the data by keeping $a=0.1$ and treating the other three parameters to be free leading to $|\zeta|=0.65$, $\alpha=0.4$ and $\beta=0.7$. A similar analysis has also been carried out for $|\zeta|=0.70$, corresponding to the extreme value of $\zeta$, whereas $\alpha$ and $\beta$ are treated as free parameters, yielding $\alpha=0.4$ and $\beta=0.6$. The parameter $a$, as noted earlier also $[3-5,16]$, plays a very important role in carrying out the overall fit in the $\chi \mathrm{CQM}$, therefore we have attempted a fit by also keeping the parameter $a$ to be free. However, to keep the escalation of the parameters under check, we have carried out a fit wherein $a, \alpha$ and $\zeta$ are treated as free parameters whereas $\beta$ is taken to be equal to $\alpha$, in accordance with our broader analysis as well as suggested by Cheng and $\mathrm{Li}[3]$, leading to $a=0.13,|\zeta|=0.10, \alpha=\beta=0.45$. Interestingly, this fit turns out to be as good as the fit wherein all the four parameters are treated free, henceforth, this fit would be referred to as the best fit. To have a deeper understanding of the $\zeta$ values corresponding to the best fit, we have also carried out our analysis where there is no contribution of the singlet GB $(\zeta=0)$ with $a, \alpha$ and $\beta$ being treated free, yielding the values $a=0.14, \alpha=0.4$ and $\beta=0.2$. For the purpose of discussion, even for the quark distribution functions, the $\zeta$ values corresponding to $|\zeta|=0.65$ and $|\zeta|=0.70$ would be referred to as higher values of $\zeta$ (Cases I and II respectively) whereas $\zeta=0$ and $|\zeta|=0.10$ would be referred to as lower values of $|\zeta|$. The analysis with $\zeta=0$ is referred to as Case III whereas our best fit, for the sake of uniformity, is referred to as Case IV.

In Table I, we have presented the results of our fits mentioned above. A cursory look at the table immediately brings out that our best fit clearly has much better overlap with the data compared to all other fits presented in the table. However, before getting into the detailed comparison of this fit with the data, we compare the two fits corresponding to the higher values of $|\zeta|$ with each other, for example $|\zeta|=0.65$ and $|\zeta|=0.70$, primarily for the purpose of understanding the role of $\eta^{\prime}$. The two cases expectedly are not very different from each other, however on closer examination one finds that Case I is uniformly better than II even though by a very small amount. The slight improvement observed in the cases of $\Delta u, \Delta d$ and $\Delta_{8}$ can be understood as a consequence of the change in 
the value of the parameter $\zeta$ as the above mentioned quantities can be seen to have weak dependence on $\alpha$ and $\beta$ in comparison to $\zeta$. Therefore, we tend to conclude that lowering of $|\zeta|$ leads to better overlap with data. This becomes all the more clear when one notes that in case of our best fit, the $|\zeta|$ value is 0.10 , much lower compared to $|\zeta|=0.65$ and $|\zeta|=0.70$. This is further borne out by the fact that keeping $a=0.13$ and $\alpha=\beta=0.45$, however changing $|\zeta|$ towards higher values, worsens the fit compared to our best fit, nevertheless the fit remains better compared with higher values of $|\zeta|$.

In the case of our best fit, we are able to obtain an excellent fit for $\Delta u, \Delta d$ and $\Delta_{8}$, in contrast with the other cases considered here. It needs to be mentioned that $\Delta_{8}$ cannot be fitted for "higher values" of $|\zeta|$ even after scanning the entire parameter space for $a, \alpha$ and $\beta$, suggesting that only the lower values of $|\zeta|$ are compatible with data. This can be easily understood when one closely examines the expression for $\Delta_{8}$ wherein one finds that it is completely dominated by the parameter $a$. As already observed, the parameters $a$ and $\zeta$ are related through $\bar{u}-\bar{d}$ asymmetry, therefore while fitting $\Delta_{8}$, requiring relatively higher values of $a$, leads to only lower values of $|\zeta|$. One may wonder why $\Delta_{3}(=\Delta u-\Delta d)$ gets fitted for all the fits. This can be easily understood when one realizes that even though $\Delta u$ and $\Delta d$ have significant dependence on $a$ but this dependence gets cancelled in $\Delta_{3}$. Similarly, one can understand that $\Delta_{3}$ also does not have any significant dependence on $\zeta$ because of the above mentioned cancellation.

After finding that the lower values of $|\zeta|$ giving much better overlap with data, one would like to check whether the same remain true in the case of octet magnetic moments or not. In this case also we find that our best fit values corresponding to lower value of $|\zeta|$ (Case IV) has a much better overlap with data compared to the higher values of $|\zeta|$. Specifically, in the case of $\mu_{n}, \mu_{\Sigma^{-}}, \mu_{\Sigma^{+}}$and $\mu_{\Xi^{-}}$, the fit with lower value of $|\zeta|$ scores over higher values of $|\zeta|$ in a marked manner. Again this remains true when we scan the entire parameter space. It may also be mentioned that $\mu_{\Xi^{-}}$is a difficult case in most of the quark models [31].

To understand the extent to which the value of $|\zeta|$ can be lowered, we now consider Case III which corresponds to the singlet GB being absent $(\zeta=0)$. A general look at the table indicates that in the absence of the singlet GB, even though the other parameters being kept free, we find that the data clearly indicates preference for a small non-zero value of $|\zeta|$. For example, in the case $\mu_{p}, \mu_{n}, \mu_{\Sigma^{-}}, \mu_{\Sigma^{+}}, \mu_{\Xi o}$ and $\mu_{\Xi^{-}}$, the $|\zeta|=0.10$ fit has clearly better overlap with data as compared to $\zeta=0$. In the case of $\mu_{\Xi^{-}}$, one may wonder, why such a small change in the value of $\zeta$ affects the fit in a marked manner. The significant improvement in the case of $\mu_{\Xi-}$ for the best fit, compared to the $\zeta=0$ case, can be easily understood when one realizes that the magnetic moments have been formulated by considering the valence, sea and orbital contributions with appropriate signs [16]. A small change in the value of $\zeta$ changes the sea and orbital contributions which constructively add to the total contribution, hence justifying the better agreement with data in the case of $|\zeta|=0.10$ compared to $\zeta=0$. In a similar manner one can understand the better agreement achieved here with data for the case of other magnetic moments.

In Table II, we have presented the results corresponding to quark distribution functions for different fits. In this case also the fit for the lower values of $|\zeta|$ is better as compared to the higher values in the case of $\bar{u} / \bar{d}$. It is interesting to observe that even for a small deviation in the value of $\zeta, f_{s}$ gets affected significantly, therefore a measurement of $f_{s}$ would give a very strong signal about the coupling strength of $\eta^{\prime}$ in the $\chi$ CQM. It may be mentioned that our conclusion regarding the small but non-zero value of $|\zeta|$ being preferred over $\zeta=0$ is not only in agreement with latest $\bar{u}-\bar{d}$ measurement [8] but is also in agreement with the conclusions of Ohlsson et. al. [5].

The conclusions arrived at above can perhaps be understood from a more theoretical point of view also. In the $\chi \mathrm{CQM}$, it is difficult to think of a mechanism wherein the contribution of $\eta^{\prime}$ or the singlet GB becomes zero, whereas a small value of the coupling strength of $\eta^{\prime}$ from phenomenological considerations is in agreement with the arguments advanced by S. Bass [20] and emphasized in the beginning. This is also in agreement with the suggestion of Cheng and $\mathrm{Li}[3]$ wherein they have suggested that the coupling strength of the GB corresponding to the pion, $K, \eta$ and $\eta^{\prime}$ mesons are inversely proportional to the square of their respective masses and are respectively of the order $a \alpha^{2} \sim 0.02$, $a \beta^{2} \sim 0.02$ and $a \zeta^{2} \sim 0.001$ for $a \sim 0.13$. This strangely agrees with the values obtained through our best fit as well as suggests the equality of the coupling strength of $\alpha$ and $\beta$.

In the Table I, we have also presented the results of our calculations for the flavor singlet component of the spin of proton $\Delta \Sigma$, however we have not discussed its implications for different cases. It has already been discussed earlier in $\chi \mathrm{CQM}[32]$ that $\Delta \Sigma$ receives contributions from various sources such as valence quarks, quark sea, gluon polarization etc.. In the context of $\chi \mathrm{CQM}$, it seems that gluon anomaly not only contributes to the gluon polarization but also is responsible for the large mass of $\eta^{\prime}[20]$. As a result, its contribution to $\Delta \Sigma$ gets correspondingly reduced compared to the contributions of valence quarks and quark sea. In this context, we would like to mention that the above non-zero 
but small contribution of $\eta^{\prime}$ looks to be well in agreement with our earlier calculations regarding the partitioning of the nucleon spin [32].

To summarize, with a view to phenomenologically estimating the coupling strength of the singlet Goldstone boson $\eta^{\prime}$ in $\chi \mathrm{CQM}_{\text {config, }}$, we have carried out a detailed analysis using the latest data regarding $\bar{u}-\bar{d}$ asymmetry, the spin

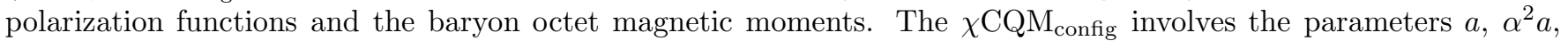
$\beta^{2} a$ and $\zeta^{2} a$, representing respectively the probabilities of fluctuations to pions, $K, \eta$ and $\eta^{\prime}$, as well as $\phi$ which is fixed from the consideration of neutron charge radius $[12,23,29]$. As a first step of the analysis, we have found from broad considerations the required ranges of these parameters using the data pertaining to $\Delta u, \Delta_{3}, \bar{u}-\bar{d}, \bar{u} / \bar{d}$ etc.. The ranges obtained are $0.10 \lesssim a \lesssim 0.14,0.2 \lesssim \alpha \lesssim 0.5,0.2 \lesssim \beta \lesssim 0.7$ and $0.10 \lesssim|\zeta| \lesssim 0.70$. After obtaining the ranges, analysis has been carried out corresponding to four different sets of the $\chi$ CQM parameters within the ranges mentioned above as well as keeping in mind the earlier analyses done in this regard. In the first case, the pion fluctuation parameter $a$ is taken as 0.1 , the value considered by several earlier analyses [3-5,16], whereas $\Delta u, \Delta_{3}$, $\bar{u}-\bar{d}, \bar{u} / \bar{d}$ etc. are fitted by treating the other three parameters to be free. This analysis yields $|\zeta|=0.65, \alpha=0.4$ and $\beta=0.7$ and is referred to as Case I. A similar analysis has also been also been carried out by taking $a=0.1$ and $|\zeta|=0.70$ whereas the parameters $\alpha$ and $\beta$ are treated free, yielding $\alpha=0.4$ and $\beta=0.6$ and is referred to as Case II. Our best fit is obtained by varying $a, \zeta$ and $\alpha$, the parameter $\beta$ is taken to be equal to $\alpha$, in accordance with the suggestions of Cheng and $\operatorname{Li}[3]$. Interestingly, this fit is not affected when $\alpha$ and $\beta$ are not taken equal and the best fit values of the parameters are $a=0.13,|\zeta|=0.10, \alpha=\beta=0.45$. We have also carried out a fit where there is no contribution of the singlet GB $(\zeta=0)$ and $a, \alpha$ as well as $\beta$ are treated free, yielding $a=0.14, \alpha=0.4$ and $\beta=0.2$. The analysis with $\zeta=0$ is referred to as Case III whereas the best fit is referred to as Case IV. The values of the parameter corresponding to $|\zeta|=0.65$ and $|\zeta|=0.70$ are referred to as higher values of $|\zeta|$ whereas those corresponding to $\zeta=0$ and $|\zeta|=0.10$ are referred to as lower values of $|\zeta|$. A comparison of all the fits clearly shows that our best fit is not only better than other fits carried out here but also provides an excellent overall fit to the data particularly in the case of $\Delta u, \Delta d, \Delta_{8}, \mu_{n}, \mu_{\Sigma^{-}}, \mu_{\Sigma^{+}}, \mu_{\Xi^{\circ}}$ and $\mu_{\Xi^{-}}$.

As already discussed in detail, comparison of Cases I and II suggest that in general lower values of $|\zeta|$ are preferred over higher values of $|\zeta|$. This is borne out clearly by a closer look at our best fit. In fact, it is interesting to mention that any change of $|\zeta|$ from the value 0.10 , worsens the fit. This can further be understood very easily by analyzing $\bar{u}-\bar{d}$ asymmetry, $\Delta_{8}$ as well as $\mu_{n}, \mu_{\Sigma^{-}}$and $\mu_{\Xi^{-}}$. The analysis of $\bar{u}-\bar{d}$ asymmetry clearly suggests that the values of $a$ and $\zeta$ are strongly coupled to each other independent of the values of $\alpha$ and $\beta$. Further, the parameter $a$ plays the most important role in fitting the polarization functions $\Delta u, \Delta d, \Delta_{3}$ and $\Delta_{8}$ whereas the role of $\alpha$ and $\beta$ is much less significant. Thus, one finds that the higher values of $a$ have to go with the lower values of $|\zeta|$ and vice-versa. Unlike the Case I, in the best fit we are able to fit $\Delta u, \Delta d, \Delta_{3}$ and $\Delta_{8}$ simultaneously. Similar conclusion can be arrived at from an analysis of the magnetic moments $\mu_{n}, \mu_{\Sigma^{-}}, \mu_{\Sigma^{+}}$and $\mu_{\Xi^{-}}$. From the above discussion, one can conclude that the data very strongly suggests that lower values of $|\zeta|$ and correspondingly higher values of $a$ are preferred over the higher values of $|\zeta|$ and lower values of $a$.

To further understand the coupling strength of $\eta^{\prime}$ in $\chi \mathrm{CQM}$, we have carried out in Case III an analysis where the contribution of $\eta^{\prime}$ is taken to be zero. Interestingly, we find that the fit obtained in this case cannot match our best fit even if the other parameters are treated completely free, suggesting that the $\zeta=0$ case can be is excluded phenomenologically. This conclusion regarding the exclusion of the singlet GB also looks to be in agreement with the theoretical considerations based on the arguments of Cheng and $\mathrm{Li}$ [3] and those of $\mathrm{S}$. Bass [20]. It seems that the phenomenological analyses of spin polarization functions, quark distribution functions and baryon octet magnetic moments, strongly suggest a small but non-zero value of $|\zeta|$ within the dynamics of chiral constituent quark model, suggesting an important role for $\eta^{\prime}$ in the non-perturbative regime of QCD. This fact perhaps can be substantiated by a measurement of the quark distribution function $f_{s}$ which shows a strong dependence on the value of $\zeta$.

\section{ACKNOWLEDGMENTS}

H.D. would like to thank DST (OYS Scheme), Government of India, for financial support and the chairman, Department of Physics, for providing facilities to work in the department. 
[1] S. Weinberg, Physica A 96, 327 (1979); A. Manohar and H. Georgi, Nucl. Phys. B 234, 189 (1984).

[2] E.J. Eichten, I. Hinchliffe and C. Quigg, Phys. Rev. D 45, 2269 (1992).

[3] T.P. Cheng and Ling Fong Li, Phys. Rev. Lett. 74, 2872 (1995); hep-ph/9709293; Phys. Rev. D 57, 344 (1998).

[4] X. Song, J.S. McCarthy and H.J. Weber, Phys. Rev. D 55, 2624 (1997); X. Song, Phys. Rev. D 57, 4114 (1998).

[5] J. Linde, T. Ohlsson and Hakan Snellman, Phys. Rev. D 57, 452 (1998); T. Ohlsson and H. Snellman, Eur. Phys. J., C 7, 501 (1999).

[6] EMC Collaboration, J. Ashman et al., Phys. Lett. 206B, 364 (1988); Nucl. Phys. B 328, 1 (1989); SMC Collaboration, B. Adeva et al., Phys. Lett. 302B, 533 (1993); P. Adams et al., Phys. Rev. D 56, 5330 (1997); E142 Collaboration, P.L. Anthony et al., Phys. Rev. Lett. 71, 959 (1993); E143 Collaboration, K. Abe et al., Phys. Rev. Lett. 75, 391 (1995); HERMES Collaboration, K. Ackerstaff et al., Phys. Lett. 404B, 383 (1997).

[7] New Muon Collaboration, P. Amaudruz et al., Phys. Rev. Lett. 66, 2712 (1991); M. Arneodo et al., Phys. Rev. D 50, R1 (1994).

[8] E866/NuSea Collaboration, E.A. Hawker et al., Phys. Rev. Lett. 80, 3715 (1998); J.C. Peng et al., Phys. Rev. D 58, 092004 (1998); R. S. Towell et al., ibid. 64, 052002 (2001).

[9] K. Gottfried, Phys. Rev. Lett. 18, 1174 (1967).

[10] A. De Rujula, H. Georgi and S.L. Glashow, Phys. Rev. D 12, 147 (1975).

[11] N. Isgur, G. Karl and R. Koniuk, Phys. Rev. Lett. 41, 1269 (1978); N. Isgur and G. Karl, Phys. Rev. D 21, 3175 (1980); N. Isgur et al., Phys. Rev. D 35, 1665 (1987); P. Geiger and N. Isgur, Phys. Rev. D 55, 299 (1997).

[12] A. Le Yaouanc, L. Oliver, O. Pene and J.C. Raynal, Phys. Rev. D 12, 2137 (1975); ibid.. 15, 844 (1977).

[13] L.Ya. Glozman and D.O. Riska, Phys. Rep. 268, 263 (1996), L.Ya. Glozman, Z. Papp and W. Plessas, Phys. Lett. 381B, 311 (1996).

[14] T.P. Cheng and Ling Fong Li, hep-ph/9811279.

[15] Adam P. Szczepaniak and Erie S. Swanson Phys. Rev. Lett. 87, 072001 (2001).

[16] H. Dahiya and M. Gupta, Phys. Rev. D 64, 014013 (2001); ibid. D 66, 051501(R) (2002); ibid. D 67, 074001 (2003); ibid. 67, 114015 (2003).

[17] T.P. Cheng and Ling Fong Li, Phys. Rev. Lett. 80, 2789 (1998).

[18] J. Franklin, Phys. Rev. 182, 1607 (1969).

[19] S. Weinberg, Phys. Rev. D 11, 3583 (1975); G 't hooft, Phys. Rev. Lett. 37, 8 (1976); Phys. Rev. D 14, 3432 (1976); R. Jackiw and C. Rebbi, Phys. Rev. Lett. 37, 172 (1976); C.G. Callan, R.F. Dashen and D.J. Gross,Phys. Lett. 63B, 334(1976); R.J. Crewther, Phys. Lett. 70B, 349 (1977); M. Ida, Prog. Theor. Phys. 61 618, 1784 (1979); Y. Hosotani, Prog. Theor. Phys. 61, 1452 (1979); R.J. Crewther, Riv. Nuovo. Cim. 2, 8 (1979).

[20] Steven D. Bass, Phys. Lett. 463B, 286 (1999); Czech. J. Phys. 50, 109 (2000); Nucl. Phys. Proc. Suppl. 105, 56 (2002); hep-ph/0411005.

[21] E. Witten, Nucl. Phys. B 156, 269 (1979); G. Veneziano, Nucl. Phys. B 159, 213 (1979).

[22] K. Kawarabayashi and N. Ohta, Nucl. Phys. B 175, 477 (1980); Prog. Theor. Phys. 66, 1789 (1981); H. Hata, T. Kugo and N. Ohta, Nucl. Phys. B 178, 527 (1981).

[23] P.N. Pandit, M.P. Khanna and M. Gupta, J. Phys. G 11, 683 (1985).

[24] M. Gupta and N. Kaur, Phys. Rev. D 28, 534 (1983); M. Gupta, J. Phys. G: Nucl. Phys. 16, L213 (1990).

[25] A. Le Yaouanc et al., Hadron Transitions in the Quark Model, Gordon and Breach, 1988.

[26] J.D. Bjorken, Phys. Rev. 148, 1467 (1966); Phys. Rev. D 1, 1376 (1970).

[27] J. Ellis and R.L. Jaffe, Phys. Rev. D 9, 1444 (1974); ibid. 10, 1669 (1974).

[28] NA51 Collaboration, A. Baldit et al., Phys. Lett. 253B, 252 (1994).

[29] M. Gupta and A.N. Mitra, Phys. Rev. D 18, 1585 (1978); N. Isgur, G. Karl and D.W.L. Sprung, ibid 23, 163 (1981).

[30] S. Eidelman et al., Phys. Lett. B 592, 1 (2004).

[31] D.B. Lichtenberg, Z. Phys. C 7, 143 (1981); ibid. 17, 57 (1983); S. Theberge and A.W. Thomas, Phys. Rev. D 25, 284 (1982); J. Cohen and H.J. Weber, Phys. Lett. 165B, 229 (1985); D.A. Dicus et al., Phys. Lett. 284B, 384 (1992); L. Brekke, Ann. Phys. (N.Y.) 240, 400 (1995); K. Dannbom, L. Ya. Glozman, C. Helminen, D.O. Riska, Nucl. Phys. A616, 555 (1997); J. Franklin, Phys. Rev. D 61, 098301 (2000).

[32] H. Dahiya and M. Gupta, Int. Jol. of Mod. Phys. A, Vol. 19, No. 29, 5027 (2004).

[33] A.O. Bazarko et al., Z. Phys C 65, 189 (1995); J. Grasser, H. Leutwyler and M.E. Saino, Phys. Lett. 253B, 252 (1991); S.J. Dong, J.F. Lagae, K.F. Liu, Phys. Rev. Lett. 75, 2096 (1995). 


\begin{tabular}{|c|c|c|c|c|c|}
\hline \multirow[t]{5}{*}{ Parameter } & \multirow[t]{5}{*}{ Data } & \multicolumn{4}{|c|}{$\chi \mathrm{CQM}_{\text {config }}$} \\
\hline & & Case I & Case II & Case III & Case IV \\
\hline & & $|\zeta|=0.65$ & $|\zeta|=0.70$ & $\zeta=0$ & $|\zeta|=0.10$ \\
\hline & & $a=0.1$ & $a=0.1$ & $a=0.14$ & $a=0.13$ \\
\hline & & $\beta=0.7$ & $\beta=0.6$ & $\beta=0.2$ & $\beta=0.45$ \\
\hline$\Delta u$ & $0.85 \pm 0.05[6]$ & 0.947 & 0.955 & 0.925 & 0.913 \\
\hline$\Delta d$ & $-0.41 \pm 0.05[6]$ & -0.318 & -0.312 & -0.352 & -0.364 \\
\hline$\Delta_{3}$ & $1.267 \pm 0.0035[30]$ & 1.267 & 1.267 & 1.267 & 1.267 \\
\hline$\Delta_{8}$ & $0.58 \pm 0.025[30]$ & 0.67 & 0.68 & 0.61 & 0.59 \\
\hline$\Delta \Sigma$ & $0.19 \pm 0.025[30]$ & 0.31 & 0.31 & 0.28 & 0.27 \\
\hline$\mu_{p}$ & $2.79 \pm 0.00[30]$ & 2.80 & 2.80 & 2.83 & 2.81 \\
\hline$\mu_{n}$ & $-1.91 \pm 0.00[30]$ & -1.99 & -2.00 & -2.16 & -1.96 \\
\hline$\mu_{\Sigma^{-}}$ & $-1.16 \pm 0.025[30]$ & -1.20 & -1.21 & -1.32 & -1.19 \\
\hline
\end{tabular}

TABLE I. The calculated values of the spin polarization functions and baryon octet magnetic moments for different cases. The value of the mixing angle $\phi$ is taken to be $20^{\circ}$.

\begin{tabular}{|c|c|c|c|c|c|}
\hline \multirow[t]{5}{*}{ Parameter } & \multirow[t]{5}{*}{ Data } & \multicolumn{4}{|c|}{$\chi \mathrm{CQM}$} \\
\hline & & Case I & Case II & Case III & Case IV \\
\hline & & $|\zeta|=0.65$ & $|\zeta|=0.70$ & $\zeta=0$ & $|\zeta|=0.10$ \\
\hline & & $a=0.1$ & $a=0.1$ & $a=0.14$ & $a=0.13$ \\
\hline & & $\beta=0.7$ & $\beta=0.6$ & $\beta=0.2$ & $\beta=0.45$ \\
\hline $\bar{u}$ & - & 0.168 & 0.167 & 0.250 & 0.233 \\
\hline $\bar{d}$ & - & 0.288 & 0.293 & 0.366 & 0.350 \\
\hline $\bar{u}-\bar{d}$ & $-0.118 \pm 0.015[8]$ & -0.120 & -0.127 & -0.116 & -0.117 \\
\hline $\bar{u} / \bar{d}$ & $0.67 \pm 0.06[8]$ & 0.58 & 0.57 & 0.68 & 0.67 \\
\hline$I_{G}$ & $0.254 \pm 0.005$ & 0.253 & 0.248 & 0.255 & 0.255 \\
\hline$f_{u}$ & - & 0.655 & 0.654 & 0.677 & 0.675 \\
\hline$f_{d}$ & - & 0.442 & 0.445 & 0.470 & 0.466 \\
\hline$f_{s}$ & $0.10 \pm 0.06[33]$ & 0.061 & 0.058 & 0.039 & 0.039 \\
\hline
\end{tabular}

TABLE II. The calculated values of the quark flavor distribution functions for different cases. 